\title{
LA “NOCHE OSCURA DEL ALMA" Y EXTERNALISMO SOBRE LA FE
}

Begoña Pessis García Instituto de Filosofía, Pontificia Universidad Católica de Chile

bpessis@uc.cl

José Tomás Alvarado Marambio Instituto de Filosofía, Pontificia Universidad Católica de Chile jose.tomas.alvarado@gmail.com

\section{Resumen}

Se han presentado recientemente diferentes propuestas acerca de la naturaleza de la fe. En muchas de ellas no hay una comprensión y una discusión suficientes de varios fenómenos que le ocurren a algunas personas con una vida interior de fe profunda. Uno de estos fenómenos es la "noche oscura del alma" - tal como fue denominado por san Juan de la Cruz-. En una noche oscura la presencia de Dios se oscurece para el fiel; de hecho, le parece que no tiene fe. Ni las teorías internalistas de la fe, ni las teorías no cognitivistas de la fe parecen aptas para explicar adecuadamente cómo es posible un fenómeno como la noche oscura. En cambio, las teorías de la fe cognitivistas y externalistas parecen apropiadas.

Palabras clave: cognitivismo, externalismo, fe sobrenatural, internalismo, noche oscura. 


\section{The ‘Dark Night of the Soul’ and Externalism on Faith}

Abstract

Different proposals have been presented recently about the nature of faith. In many of these theories there has not been a sufficient understanding and discussion of several phenomena that occurs to people with a deep interior life of faith. One of these phenomena is the noche oscura del alma -as it was dubbed by Saint John of the Cross. In a noche oscura the presence of God is darkened for the faithful. In fact, it seems to him that he has no faith. Neither internalist theories of faith, nor non-cognitivist theories of faith seem able to explain properly how it is possible a phenomenon like la noche oscura. Instead, cognitivist and externalist theories of faith seem appropriate.

Keywords: Cognitivism, Dark Night of the Soul, Externalismo, Internalism, Supernatural Faith. 
En tiempos recientes ha aparecido una serie de propuestas teóricas para comprender la naturaleza de la fe en filosofía de la religión. Por un lado, se han presentado teorías para las que la fe es un tipo de estado cognitivo especial (Swinburne, 2003; Plantinga, 1983, 2000; Alston, 1993) en el que se posee cierto conocimiento o creencia justificada. Por otra parte, se han intentado desarrollar teorías en las que la fe tiene que ver más bien con algún estado emocional o afectivo, o bien con un estado volitivo de "resolución interior" (Bishop, 2007, 2010). La disputa fundamental entre estas alternativas tiene que ver con el carácter de conocimiento o de justificación que la fe pueda tener. Esto está conectado, además, con cuestiones más generales de epistemología de la religión y teología natural. Por ejemplo, es posible estar más inclinado a sostener que la fe tiene que ver ante todo con componentes afectivos y volitivos o bien estar más inclinado a otorgarle un valor epistemológico. En uno o en otro caso, sin embargo, se ha desatendido - crucialmente- la experiencia de vida concreta de fe de los fieles. A veces pareciera que los filósofos - tal vez por un afán de simplificar y clarificar el análisis atendiendo a ciertos 'tipos ideales' - trabajan pensando en un estereotipo acerca de aquello en lo que consista tener fe y sobre cómo es la vida de fe de una persona. Este trabajo tiene por objetivo corregir, en parte, esta desatención, o - si se quiere- contribuir al desarrollo de un tratamiento más equilibrado en este punto.

El fenómeno de la "noche oscura del alma" es un caso de análisis especialmente importante e ilustrativo de estas desatenciones. Se trata de un fenómeno muy documentado que le ocurre a personas con mucha vida interior de oración, que están fuertemente comprometidas en vivir su fe y que han logrado configurar un carácter moral definido en conformidad con estas resoluciones interiores. Sucede a personas cuya vida ha sido presentada por la Iglesia Católica como un ejemplo de virtudes cristianas puestas en obra y, en particular, de fe, esperanza y caridad. Se trata, por lo tanto, de un tipo de fenómeno de especial importancia para comprender la naturaleza de la fe.Y, tal como se hará ver, hay tesis sustantivas que parecen tener aptitud explicativa para tratar este fenómeno, mientras que hay otras que no la tienen. En especial, se va a sostener aquí que una consideración 
atenta de la "noche oscura del alma" parece obligar a algún tipo de teoría externalista de la fe.

Se ha hecho ya notar la importancia del fenómeno de la noche oscura del alma en filosofía de la religión, pero atendiendo a la cuestión del "ocultamiento de Dios" (Howard-Snyder y Moser, 2002; Moser 2008) y no a la cuestión de la naturaleza de la fe y de su valor epistemológico. Lo que interesa aquí, en cambio, es contestar la pregunta acerca de qué es la fe y considerar qué tipo de restricciones impone el fenómeno de la noche oscura para comprender su naturaleza.

En lo que sigue, por lo tanto, se harán en la primera sección algunas precisiones conceptuales. Luego, se explicará en la segunda sección la naturaleza de la llamada "noche oscura del alma". En la tercera sección se explicarán los problemas que el fenómeno de la noche oscura del alma genera para las teorías internalistas y no cognitivistas. En la cuarta sección, por último, se presentará un modelo diferente, externalista, donde el fenómeno de la "noche oscura" es explicable.

\section{Precisiones conceptuales}

En discusiones temáticas de este tipo, ante la inexcusable pregunta inicial “¿qué es la fe?", puede ser útil considerar la caracterización católica tradicional. En el capítulo 3 de la Constitución Dogmática Dei Filius del Concilio Vaticano I del 24 de abril de 1870, se señala que la fe es una "virtud sobrenatural por la que, con inspiración y ayuda de la gracia de Dios, creemos ser verdadero lo que por él ha sido revelado, no por la intrínseca verdad de las cosas, percibida por la luz natural de la razón, sino por la autoridad del mismo Dios que revela, el cual no puede ni engañarse ni engañarnos" (DH 3008; cf. los cánones 1 y 2, DH 3031 y 3032, respectivamente; también, Pío IX, Encíclica Qui Pluribus de 9 de noviembre de 1846, en DH 2778). Una virtud es una propiedad disposicional de un sujeto racional y libre por la que pueden realizar acciones moralmente buenas de cierto tipo con 'facilidad'. La virtud sobrenatural de la fe se especifica porque dispone para creer ser verdadero lo que ha sido revelado por 
Dios. El acto de fe sobrenatural es, entonces, el acto de creer ser verdadero lo que ha sido revelado por Dios, por la autoridad del mismo Dios que revela, el cual no puede engañarse ni engañarnos.

Es característico de esta concepción católica el que la fe sea tenida por una creencia con un objeto proposicional, que es aquello que se cree, y cuyo motivo es la revelación que Dios ha hecho de tal verdad (Tomás de Aquino, ST II-II, q. 1, aa. 7-9). Una "creencia” es una actitud proposicional por la que se acepta una proposición como verdadera, sea que el sujeto de tal creencia tenga una justificación adecuada para pensar que la proposición en cuestión es verdadera, sea que tenga una justificación inadecuada, o sea que no tenga ninguna justificación. La creencia de que $p$ es neutral, por lo tanto, en cuanto a si $p$ es verdadera o falsa, y en cuanto a si el sujeto de la creencia está epistemológicamente legitimado para adoptarla. Se trata, en efecto, de una actitud proposicional, pues el objeto del estado mental es una proposición, que es aquello que es creído y que es portador de un valor de verdad. ${ }^{1}$ La justificación que ha de tener el sujeto de la creencia para adoptarla, si es que se trata realmente de un caso de fe sobrenatural, es la revelación de Dios, quien posee autoridad epistémica máxima - "no puede engañarse ni engañarnos". La fe sobrenatural se presenta como un caso de justificación por testimonio en el que la fe o confianza en quien asevera la proposición que va a ser objeto de aceptación es el motivo por el que uno resulta epistemológicamente legitimado para creer tal proposición. El acto de habla de aseveración por el que el testigo enuncia la proposición objeto de fe se ha denominado "revelación". En el caso de la fe sobrenatural, el testigo es, por supuesto, Dios mismo. ${ }^{2}$

1 La forma estándar en que describimos los estados mentales de un sujeto es mediante la indicación de qué actitud tiene este sujeto respecto de diferentes proposiciones que -se supone- ese sujeto puede comprender.

2 En la concepción católica, entonces, la fe sobrenatural es "sobrenatural" debido a que el testigo de la verdad de las proposiciones objeto de fe es Dios mismo. No es "sobrenatural" porque se requiera un auxilio especial de la gracia para este asentimiento, aunque nada de lo que se diga aquí obste para asignarle una función fundamental a la gracia (cf. Constitución Dogmática Dei Filius del Concilio Vaticano I, canon 5, DH 3035). Tampoco es "sobrenatural" porque las proposiciones objeto de fe estén haciendo atribuciones a una entidad que se encuentra por encima de las realidades 'naturales', esto es, Dios. 
Es importante destacar que en esta concepción la fe sobrenatural se presenta como una forma de conocimiento. El 'conocimiento' puede ser caracterizado - sin necesidad de entrar a prejuzgar ninguna de las cuestiones de fondo debatidas en la epistemología de los últimos cincuenta años- como el estado mental factivo más general (Williamson, 2000: p. 27-48). Un estado mental $\Phi$ respecto de una proposición $p$ es factivo si y sólo si, si el sujeto $S \Phi$ que $p$, entonces $p$. Esto es, el objeto de tal estado de mental debe tomarse como un hecho (factum). El conocimiento es el estado mental factivo más general porque, (i) si $S$ conoce que $p$, entonces $p$-esto es, el conocimiento es un estado mental factivo-y, (ii) si $\Phi$ es un estado mental factivo, entonces si $S \Phi$ que $p$, entonces $S$ conoce que $p$. Si un tipo de estado mental es factivo en los términos indicados, entonces se trata de un tipo de conocimiento. ${ }^{3}$ La fe sobrenatural se presenta aquí como un estado mental factivo porque las proposiciones sobre las que versa son - de entrada-verdades reveladas por Dios. Sucede, en efecto, que si $S$ tiene fe sobrenatural de que $p$ entonces $p$, pues la proposición objeto de fe no podría no ser verdadera (cf. Lumen fidei, nn. 23-36). Si no lo fuese, entonces no se trataría realmente de un caso de fe sobrenatural, tal como si alguien pensase que $p$ y $p$ fuese falsa, entonces no tendría conocimiento de que $p$, no impor ta lo buena que pueda ser la justificación que tuviese para pensar que $p$.

Ésta es una concepción cognitiva de la fe sobrenatural, en donde se trata de una actitud proposicional con valor epistemológico. Tal como se ha indicado más arriba, se han defendido recientemente otras teorías cognitivas de la fe. Alvin Plantinga y los restantes defensores de la llamada "epistemología reformada" han sostenido que poseemos creencias básicas dotadas de "garantía” (warrant) acerca de Dios (Plantinga, 1983, 2000; Alston, 1993). En la teoría epistemológica general de Plantinga, la "garantía" es lo que se requiere para que una creencia cuente como conocimiento (Plantinga, 1993b: pp. 3-47; 1993a: pp. 43-46, pp. 51-65). Sostener, por lo tanto, que

3 Son casos de conceptos de estados mentales factivos, por ejemplo, "ver", "oír" o "recordar", al menos, tal como se utilizan ordinariamente. Si "ver" es, entonces, un estado mental factivo -pues si uno ve que $p$, entonces $p$ - entonces "ver" designa una forma específica de conocer. 
poseemos creencias básicas dotadas de garantía acerca de Dios es sostener que tenemos conocimiento acerca de Dios. Se trataría de creencias "básicas" pues no son creencias que se encuentren justificadas por vínculos inferenciales con otras proposiciones que sean objeto de creencia, tal como las creencias perceptivas no se adquieren por inferencia a partir de otras creencias. Una creencia básica cuenta como conocimiento, al poseer una "garantía", si y sólo si ha sido generada por la operación de una facultad cognitiva funcionando adecuadamente en el ambiente para el que ha sido diseñada. Y una facultad cognitiva funciona adecuadamente si y sólo si esta facultad está diseñada para alcanzar la verdad y hay una probabilidad objetivamente alta de que las creencias generadas en virtud de la operación de tal facultad sean verdaderas (Plantinga, 1993b: pp. 3-20). Se trata de una teoría externalista del conocimiento y la justificación, tal como se explicará más abajo. Lo que resulta fundamental para lo que interesa aquí es que hay una facultad cognitiva especial para generar creencias verdaderas acerca de Dios si funciona adecuadamente. Siguiendo a Calvino, Plantinga ha designado a esta facultad un sensus divinitatis (Plantinga, 2000: pp. 167-198). Para las creencias específicamente cristianas lo que opera es la acción directa del Espíritu Santo que "enseña internamente" sobre las verdades reveladas a los fieles (Plantinga, 2000: pp. 241-289).

Otra importante teoría cognitivista de la fe es la que ha sido desarrollada por Richard Swinburne. Swinburne está consciente de las diferencias entre una concepción de la fe como un tipo de actitud proposicional de mera creencia y otras formas de fe en que debe admitirse un componente adicional de "compromiso" a actuar en conformidad con las creencias aceptadas bajo un sentimiento de "confianza" en Dios. El primer modelo es denominado por Swinburne "tomista” y el segundo "luterano" (cf. Swinburne, 2003, 138-147). En ambos casos la fe es, en su núcleo, una actitud proposicional de creencia. La diferencia es que en el modelo luterano no basta el aspecto cognitivo de creencia sin un correlato volitivo y afectivo adicional. Es notorio, sin embargo, que la fe tiene que ver, por lo menos, con la creencia en ciertas proposiciones -las de los símbolos de la fe-cualquiera sea la forma en que uno ha llegado a 
estar justificado en la aceptación de tales creencias. Uno posee una justificación racional para creer que $p$ dada la evidencia $e$ si y sólo si la probabilidad epistémica de $p$ condicional en la evidencia $e$ es mayor que 0,5. Una condición necesaria para tener fe-dejando a un lado los requerimientos adicionales de modelos "luteranos"- es que uno esté justificado racionalmente para creer el símbolo de la fe. No interesa la forma en que se haya obtenido tal evidencia. No importa si se ha obtenido por testimonio, tal como lo supone la teoría católica tradicional, por evidencia empírica directa o por alguna forma de inferencia. Swinburne ha argumentado extensamente, por ejemplo, a favor de la existencia de Dios y de sus principales atributos mediante una argumentación probabilística acumulativa por la que se defiende que es más racional creer que Dios existe a creer que no existe (Swinburne, 2004). Si alguien movido por esta argumentación llega a creer que Dios existe, entonces esto será, al menos en parte, constitutivo de la fe en tal sujeto. Por supuesto, requerirá también asignar una probabilidad epistémica positiva a los restantes artículos de la fe, pero no interesa la fuente de la justificación, sino lo que está siendo justificado. La teoría de Swinburne, por lo demás, difiere en un aspecto crucial respecto de la epistemología reformada, pues es internalista, de acuerdo a lo que se explicará. Las creencias básicas dotadas de "garantía" que para Plantinga constituyen conocimiento, no lo constituyen para Swinburne. La evidencia de que se trate debe resultar "accesible" al sujeto que la posee (Swinburne, 2001: pp. 152-158; pp. 206-211).

Hay también una pléyade de teorías no cognitivistas de la fe. En algunas de estas teorías la fe involucra una actitud proposicional de creencia, en otras no. En ninguna de ellas, sin embargo, se espera algún valor epistemológico para la fe. La fe no tiene que ver con la existencia de un tipo especial de justificación - tal como sucede en la teoría católica y en la epistemología reformada- o en la posesión de justificación para ciertas proposiciones específicas - tal como sucede en la teoría de Swinburne. Las teorías no cognitivistas de la fe parten de la base de que no hay justificación racional epistémica para creer las proposiciones que integran los símbolos de la fe. La naturaleza de la fe no puede buscarse, entonces, en un tipo especial 
de justificación o de evidencia, o de proposiciones para las que se tiene justificación o evidencia. Probablemente la propuesta reciente en este sentido más desarrollada es la de John Bishop de un "fideísmo moderado"(Bishop, 2007), la cual se enmarca en un "modelo pragmatista" de la consideración de la fe. Sostiene Bishop que la evidencia epistémica es neutral tanto a favor de las proposiciones del símbolo de la fe como para sus negaciones. Esto no impide, sin embargo, que pueda estar "prácticamente justificada" una "empresa doxástica” (doxastic venture) (Bishop, 2007, 101-150).

Tal como se ha indicado más arriba, el fenómeno de la noche oscura parece ofrecer dificultades especiales a teorías internalistas de la fe y parece favorecer, en cambio, teorías externalistas. Convendrá aclarar, entonces, el contraste entre "internalismo" y "externalismo". Estas expresiones se han usado de varios modos en varios contextos. Para lo que interesa aquí, sin embargo, la distinción tiene que ver con la accesibilidad de la evidencia para el sujeto que la posee (BonJour, 2002). En una concepción internalista de la justificación y del conocimiento, si alguien tiene conocimiento de que $p$ entonces el hecho de poseer tal conocimiento debe resultar accesible para él desde su perspectiva de primera persona. Del mismo modo, si tiene alguna justificación para creer que $p$, el hecho de poseerla debe resultarle transparente. Así, si un sujeto $S$ conoce que $p$ entonces $S$ debe conocer que conoce que $p$. Si $S$ tiene una justificación para creer que $p$, entonces $S$ debe estar justificado en creer que tiene una justificación para creer que $p$.

Tradicionalmente se ha supuesto que el internalismo acerca del conocimiento y de la justificación se encuentra fundado en la transparencia que deben tener todos los estados mentales para su poseedor. Un estado mental, después de todo, designa precisamente aquellos estados en que se encuentra un sujeto respecto de los que sólo él es autoridad incorregible. Sólo el sujeto que posee un estado mental puede conocer con completa certeza que lo posee. Del mismo modo, se supone que si un sujeto posee un estado mental, entonces debe conocer que posee tal estado mental. Nadie podría tener un estado mental sin saber que tiene ese estado. Poseer cierta evidencia, ya sea que sea suficiente o no para constituir conocimiento, es un 
tipo de estado mental. Luego, si uno tiene cierta evidencia uno debe saber que tiene tal evidencia. En todas las concepciones cognitivistas de la fe ésta consiste, al menos en parte, en una actitud proposicional con valor epistemológico al estar - de un modo u otro- justificada. Un internalista debería, por lo tanto, sostener que la posesión de fe debería ser algo perfectamente transparente para el sujeto que la posee. En las teorías no cognitivistas, la fe no posee valor epistemológico y puede o no ser entendida como acompañada o constituida por una actitud proposicional. Cualquiera que sea esta actitud, ella también debería resultar perfectamente transparente para el sujeto que la posee. También lo deberían ser los estados afectivos y volitivos constitutivos de la fe, cualquiera que sea la importancia que se les otorgue.

El internalismo ha sido la posición obvia en epistemología desde Descartes y por buena parte del siglo pasado. Parece ser la concepción más razonable que uno debería adoptar si es que la evidencia que pueda tener un sujeto debería ser relevante para que este sujeto pueda satisfacer sus responsabilidades epistémicas. Sin embargo, no parece una teoría adecuada para caracterizar los estados epistémicos en que puedan encontrarse sujetos poco sofisticados, con poca capacidad reflexiva o con ninguna capacidad reflexiva. No parece razonable suponer, por ejemplo, que un animal posee el concepto de "conocimiento". Ningún animal podría, por lo tanto, conocer que tiene conocimiento. Sin embargo, es obvio que los animales superiores tienen conocimiento. Lo mismo sucede con niños pequeños. El internalismo parece requerir un nivel de reflexividad exorbitante para sujetos como nosotros, con capacidades cognitivas finitas (Goldman, 1999). Por esto, parece dejar nuestras prácticas epistémicas vulnerables al escepticismo. En las concepciones externalistas se relaja el requerimiento de completa reflexividad. Uno puede tener conocimiento y, sin embargo, no es necesario que uno conozca que tiene conocimiento. De un modo semejante, uno puede tener una justificación para creer que $p$ y no conocer o no estar justificado en creer que uno tiene una justificación para creer que $p$. Por ejemplo, para el externalista uno puede tener una creencia perceptiva justificada si es que las facultades cognitivas que se han ejercido son objetivamente 
confiables, esto es, si es que es objetivamente probable que su operación genere creencias verdaderas. No es necesario que el sujeto conozca o tenga creencias bien justificadas acerca del carácter confiable de sus capacidades cognitivas. Basta con que sean confiables.

Entre las teorías que han sido mencionadas aquí, la teoría de Plantinga es claramente externalista. La existencia de fe depende solamente de que el sensus divinitatis y el Espíritu Santo estén funcionando adecuadamente, tal como él entiende la noción. No se requiere que uno conozca que están operando tales mecanismos cognitivos y que son confiables. La teoría de Swinburne es claramente internalista. Toda justificación epistemológicamente aceptable para Swinburne debe ser "internamente" accesible al sujeto que la posee. Entre las teorías no cognitivistas no hay una única posición clara al respecto, pero parte de la motivación de Bishop para su "fideísmo moderado" es la operación de exigencias internalistas para la justificación racional de las creencias que uno posea (Bishop, 2007: pp. 26-100). En el caso de la teoría católica de la fe, en donde ésta está fundada en testimonio, caben alternativas internalistas y externalistas. Depende del modo en que sea tratado el testimonio como mecanismo de justificación. En lo que sigue se van a considerar estas diferentes alternativas para concebir la naturaleza de la fe sobrenatural, no porque sean las únicas teorías existentes, sino por ser las más recientes o las más destacadas.

\section{La noche oscura del alma o noche pasiva del espíritu}

Si bien es razonable pensar que a una persona que tiene una vida interior profunda le debería constar subjetivamente que posee fe, en la práctica es posible encontrar una amplia literatura que prueba la existencia de contraejemplos. No es difícil hallar testimonios de muchos santos, en los que reportan haberse sentido privados de toda fe y haber experimentado una sensación de abandono por parte de un Dios supuestamente ausente. San Juan de la Cruz, poeta emblemático del misticismo español del siglo XVI, acuña una expresión para designar este estado de vacío y desolación espiritual: "noche 
oscura del alma” o del espíritu. Dicho padecimiento, que trae consigo muchas pruebas y tribulaciones, se devela en realidad como una vía de purificación intensa que prepara a las almas maduras y avanzadas para la unión o matrimonio espiritual con Dios, esto es, para la santidad. Aunque, en efecto, el caso de san Juan de la Cruz y de su contemporánea santa Teresa de Ávila sean los más difundidos a causa de la belleza de sus composiciones artísticas, es posible encontrar un corpus mucho más vasto de insignes religiosos que atravesaron el mismo trance: san Pío de Pietralcina, san Francisco de Asís, santa Teresa del niño Jesús, san Alfonso María de Ligorio, san Benito, san Ignacio de Loyola, entre otros. La nómina de personalidades no se agota en el pasado, puesto que nuevas investigaciones informan de casos más recientes, entre los que destaca el de la beata Teresa de Calcuta.

La tradición cristiana reconoce tres fases en el camino espiritual que emprende un buen creyente dedicado a trabajar en su fe y a profundizar en su contacto íntimo con Dios. Estas “estaciones” han sido conocidas comúnmente como las tres edades de la vida espiritual: la vía purgativa, la vía iluminativa y la vía unitiva; estas corresponden, respectivamente, a la de los principiantes o incipientes, a la de los proficientes, aprovechados o avanzados y a la de los perfectos (Garrigou-Lagrange, 1944: p. 259). En aras de esbozar una descripción de la noche oscura del alma, es menester, en primer lugar, distinguirla de otro peldaño característico de la ascendente vida espiritual casi homónimo: la "noche oscura de los sentidos"; ésta se trata de una purgación no voluntaria, por ello pasiva, de los sentidos externos e internos, de las pasiones, la inteligencia, la memoria y la voluntad. Este trance prepara el alma para el siguiente estadio espiritual: la contemplación infusa de Dios (Garrigou-Lagrange, 1944: pp. 585610; Tanquerey, 1930: 1420-1434). Pese a la notable importancia del fenómeno recién enunciado, la noche oscura que interesa detallar aquí consiste en una purificación, ya no de naturaleza sensible, sino de corte espiritual. La "noche oscura del alma" se ubica, en el mapa vertical que supone la vida interior, en una posición intermedia entre la vía iluminativa y la unitiva. Este último grado consuma y corona la trayectoria espiritual del hombre en la tierra. Antes de 
alcanzarlo, es necesario atravesar por la noche oscura; "[p]ara purificar y corregir el alma, deja Dios al entendimiento en tinieblas, a la voluntad en la sequedad, a la memoria sin recuerdos, y a los afectos perdidos en el dolor y la angustia"(Tanquerey, 1930: p. 937). Específicamente, la forma en la que esto ocurre es que el sujeto se ve acometido por fuertes y tenaces tentaciones contra las virtudes teologales de la fe, la esperanza y la caridad. Dichas virtudes conforman uno de los núcleos principales de la vida cristiana, de modo que su falta, o bien la creencia subjetiva de su falta, vuelve dificultosa y penosa la perseverancia en el camino de la santidad. No obstante, la superación exitosa de las tentaciones tiene como resultado una purificación de las virtudes teologales de las imperfecciones y defectos; de esta manera, la fe, la esperanza y la caridad se ven fortalecidas, robustecidas y renovadas mediante el destierro de todo motivo demasiado humano y sensible para practicarlas y mediante la emergencia marcada y predominante del motivo formal sobrenatural que las sostiene (Garrigou-Lagrange, 1944: pp. 977-998). En este sentido, no parece majadero insistir en que la noche oscura tiene como fin adecuar y encaminar "al alma a aquella perfecta unión con Dios que llamamos unión transformante [...] y aparece como necesaria para hacer desaparecer los defectos de los aprovechados" (Garrigou-Lagrange, 1944: p. 1089).

Aunque dicho estado esté ordenado a la santidad y a la comunión divina, los rasgos constitutivos que lo caracterizan infringen al individuo gran tormento y malestar, puesto que a este le parece que en lugar de avanzar en su encuentro con Dios, más bien retrocede y se extravía. Llevar a cabo una descripción detenida del fenómeno de la noche oscura es arduo, puesto que incluso quienes la han vivido subrayan su inefabilidad y ponen de manifiesto la dificultad que supone explicarla y hacerla inteligible para el resto. Aun así, un examen de los testimonios personales y de la literatura especializada que ha sistematizado la experiencia en cuestión hace posible identificar al menos tres características esenciales que se reconocen en toda aparición de la noche oscura, a saber, (1) el sentimiento de abandono y desamor por parte de Dios y, al mismo tiempo, (2) una sed intensa de Dios y (3) la creencia honesta de que se carece de fe sobrenatural, 
que es la que más interesa para los efectos de este trabajo. Dichos rasgos se pueden ver transparentados en un pasaje de Teresa de Calcuta en el cual se sintetiza el trinomio mencionado:

En mi corazón no hay fe-ni amor-ni confianza - hay tantísimo dolor-el dolor del anhelo, el dolor de no ser querida [(2008: p. 238)].

Estas características sintómaticas de la noche oscura no hacen sino poner al descubierto las tentaciones a las que se ven sometidos los afectados, es decir, aquellas relativas a las virtudes teologales. Las notas que se revisan a continuación representan una muestra de algunos de los modos concretos en los que se ponen a prueba dichas virtudes y, también, permiten al sujeto cultivar la auténtica virtud de la humildad.

Respecto de la primera característica mentada, es posible destacar la sensación de abandono y desamor por parte de Dios. En este momento, el individuo tiene una visión muy profunda de la fragilidad, miseria y pequeñez humana en comparación con la realidad divina. Esta conciencia hace que Dios parezca demasiado lejos del hombre y se agudiza la consideración de uno mismo como una criatura demasiado imperfecta y defectuosa. Quienes han sufrido este dolor aseguran no sentir la presencia de Dios y, en cambio, experimentarlo ausente, lejano e indiferente. San Juan de la Cruz expresa esta sensación en términos de ocultamiento divino (1994: p. 127):

¿A dónde te escondiste, Amado, y me dejaste con gemido?

Como el ciervo huiste, habiéndome herido; salí tras ti clamando, y eras ido.

Ese "sentimiento de ausencia de Dios" (Teresa de Calcuta, 2008: p. 204) implica también la impresión de que Él, voluntariamente ha decidido desaparecer. Este hecho intensifica la desesperación y la tristeza de los individuos afectados. Teresa de Calcuta se lamenta diciendo: 
Nuestro Señor pensó que era mejor para mí estar en el túnel -así Él se fue de nuevo dejándome [...] la realidad de la oscuridad y de la frialdad y del vacío es tan grande que nada mueve mi alma (2008: pp. 220-232).

Santa Teresa de Ávila nominaliza su sentimiento de pesar y desdicha a través de metáforas amorosas que describen la situación menesterosa en la que queda el alma cuando siente lejos a Dios (1994: p. 442):
Mi alma afligida
gime y desfallece.
¡Ay! ¿Quién de su Amado
puede estar ausente?

Además de las penurias del abandono, en este estado el individuo no se siente amado y protegido por Dios, sino que, por el contrario, se siente aborrecido y despreciado por Él. La madre Teresa confiesa, en su correspondencia íntima, sentir que cuanto más quiere a Dios, Él menos la quiere (2008: p. 204). El pasaje copiado a continuación es uno de los que mejor cristaliza la desesperación de la beata respecto a este punto:

En las tinieblas [...] Señor, Dios mío, ¿quién soy yo para que Tú me abandones? La niña de Tu amor-y ahora convertida en la más odiada - la que Tú has desechado como despreciada - no amada (2008: p. 231).

El segundo rasgo identificado está profundamente vinculado a los otros dos y es, precisamente, el que genera mayor tensión y dolorosa contradicción: el anhelo intenso e insaciable de Dios pese a las tribulaciones que este comporta. La agonía resultante de adolecer de incredulidad y desconfianza junto con la sensación de abandono y desamor se multiplica cuando los sujetos desean la unión y proximidad con la divinidad. La convivencia entre "una gran aridez espiritual y vivísimos deseos de la perfección” (Garrigou-Lagrange, 1944: p. 951) genera mucha inquietud y lucha interior. Teresa de 
Calcuta insiste en que "la desolación es tan grande y al mismo tiempo el anhelo por el 'Ausente' tan profundo” (2008: p. 206). Aun en el momento en que sus obras caritativas eran fecundas y su devoción inspiraba a tantas personas, la beata escribe:

Hay tanta contradicción en mi alma.- - Un deseo tan profundo de Dios - tan profundo que es doloroso - un sufrimiento continuo y sin embargo no soy querida por Dios — rechazada - vacía — ni fe — ni amor — ni fervor. - Las almas no me atraen - el Cielo no significa nada - me parece un lugar vacío - la idea del Cielo no significa nada para mí y sin embargo este atormentador anhelo de Dios. (2008: p. 211).

Santa Teresa de Ávila expresa el mismo sentimiento en sus versos (1941: p. 442-443):

Ansiosa de verte

deseo morir. $[\ldots]$

En vano mi alma

te busca, oh, mi dueño;

tú siempre invisible

no alivias su anhelo.

San Juan de la Cruz le ruega a Dios que se manifieste para calmar su espíritu contrito (1994: p. 133):

Descubre tu presencia

y máteme tu vista y hermosura;

mira que la dolencia

de amor, que no se cura

sino con la presencia y la figura.

El tercer rasgo enumerado es el que, aunque no se entiende sin la presencia de los otros, despierta más interés en el ámbito epistemológico de la posesión de la fe en los casos relativos a la "noche oscura”. El hecho paradojal que entraña este fenómeno es que los 
individuos afirman con sinceridad desconocer que tienen fe sobrenatural pese a que efectivamente la poseen. En otras palabras, los sujetos se hallan en una situación de oscuridad u opacidad epistémica respecto a su propia fe. En estos casos, el individuo afirma desconocer que tiene fe y presenta radicales dudas e inseguridades respecto de la existencia de Dios y de lo revelado por el mismo. En esta medida se entiende que "la purificación pasiva del espíritu parece consistir, [principalmente], en la ausencia de las luces anteriormente recibidas acerca de los misterios de la fe" (Garrigou-Lagrange, 1944: p. 943). La firmeza y entereza con las que solían practicar la virtud de la fe se ven amenazadas. En la noche oscura el individuo se ve privado de los motivos secundarios que ayudan al hombre a conservar su fe y a perseverar en ella; por este motivo "el acto de fe nos resulta [...] demasiado dificultoso. Lo cual acontece cuando la divina luz purificadora nos presenta lo que en estos misterios hay de más elevado y aparentemente menos conforme con la razón..." (Garrigou-Lagrange, 1944: p. 984). Pese a que la oscuridad en la que se sienten sumergidos los sujetos afectados constituya en efecto una luminosidad mucho más elevada que la que conocían anteriormente, esta luz es interpretada como noche en un primer momento dado que "una luz vivísima nos da la impresión de oscuridad en razón de su propia intensidad y de la misma alteza del objeto al cual nos conduce. Además, hácenos sufrir por causa de nuestra impureza y pequeñez, que se hace sentir más vivamente ante ciertas tentaciones del demonio, que en este período se presentan” (Garrigou-Lagrange, 1944: p. 961). En esta línea, es posible comprender por qué los autores que han padecido la noche oscura suelen emplear metáforas lumínicas para mentar la supuesta carencia de fe sobrenatural. En esta medida, el fenómeno es nominalizado como si se tratase de una "fe ciega", es decir, de una fe que no descansa en el conocimiento de sí misma y en una confianza sólida en la Revelación. En consonancia con esto, San Juan de la Cruz escribe (1994: p. 106):

Ni yo miraba cosa, sin otra luz ni guía, sino la que en el corazón ardía 
Con dichos versos el poeta afirma no poder descansar en las luces del entendimiento para que su propia fe le sea transparente y accesible en términos cognitivos. La incapacidad de ver puede interpretarse como el desconocimiento de la propia fe y la falta de luz puede referir a la sensación de abandono y de privación de auxilio divino para dicho conocimiento. Aunque algún elemento interior inclina al sujeto hacia Dios (aquello que arde en su corazón), es evidente que éste afirma desconocer que tiene una fe consciente de sí misma. Teresa de Calcuta también sostiene desconocer que tiene fe y da cuenta de la incapacidad de su entendimiento para acceder a su propia fe cuando afirma:

Sólo la fe ciega me sostiene, ya que en realidad para mí todo está en tinieblas (2008: p. 203).

En la misma línea, la beata pone al descubierto su falta de confianza y de certezas:

¿Dónde está mi fe?-Incluso en lo más profundo, todo dentro, no hay nada sino vacío y oscuridad (...) No tengo fe (...) Tantas preguntas sin respuesta viven dentro de mí- me da miedo descubrirlas - a causa de la blasfemia. - Si Dios existe, por favor perdóname. (...) En mi alma siento precisamente ese dolor terrible $(. .$.$) de que Dios realmente no existe (...) Esa oscuri-$ dad que me rodea por todas partes - no puedo elevar mi alma a Dios - no entra luz alguna ni inspiración en mi alma (2008: pp. 231-238).

Es importante notar que, en realidad, el oscurecimiento de la propia fe, que inclina a dudar de las verdades reveladas, es el rasgo más destacado de la noche oscura. Esto es así porque la fe es la base de la vida sobrenatural y de las demás virtudes, tanto de las teologales como de las morales (Tanquerey, 1930: pp. 751-763). En esta medida, es comprensible que si titubea este pilar fundamental, todo el edificio espiritual tiemble y se estremezca. En las antípodas, "el alma, que está confirmada en la fe, puede hacer frente a las 
tempestades del espíritu [... dado que la fe es] un foco de luz para el entendimiento, una fuerza y un consuelo para la voluntad" (Tanquerey, 1930: p. 756). Dado que esta característica del fenómeno analizado constituye el necesario punto de partida para que se lleve a cabo la purificación pasiva del espíritu, se recrudece la urgencia de intentar aducir una aproximación epistemológica que permita acogerla y contenerla.

\section{3. ¿Explicación internalista?}

El fenómeno de la noche oscura del alma parece estar directamente en conflicto con las concepciones internalistas de la fe. No importa si se trata de teorías cognitivistas o no cognitivistas de la fe. Esto es especialmente notorio si se considera la concepción de la fe de Swinburne: tener fe es tener creencias justificadas en las proposiciones del símbolo de la fe $-\mathrm{o}$, eventualmente, tener una creencia justificada de la conjunción de todas esas proposiciones; tener una justificación es algo que debe resultar accesible internamente para el sujeto que la posee; tener una creencia es un estado subjetivo que debe resultar también transparente para el sujeto que la posee; si alguien tiene fe, por lo tanto, debería conocer que tiene fe. ¿Cómo puede esto suceder, sin embargo, si alguien está en una etapa de noche oscura del alma? De entrada, parece que, en un caso tal como los reportados arriba, sencillamente no se tiene fe, pues no se sabe que se tiene fe. En abstracto, el internalista podría intentar un par de estrategias para resolver esta cuestión.

Una primera estrategia es sostener que realmente en los casos de noche oscura no hay fe. Esto exigiría re-describir el fenómeno de un modo bastante radical. Uno podría sostener que se trata de algo así como una patología, una suerte de histeria religiosa. Personas que por mucho tiempo han tenido una vida profunda de oración, pierden súbitamente la fe, tal vez por un burn-out debido al esfuerzo excesivo sostenido por años. Como esto sucede a personas consideradas "santas" en sus respectivas tradiciones religiosas, se ve en tales tradiciones la necesidad de explicar este proceso penoso como una 
especial purificación interior querida por Dios. Los mismos sujetos de este proceso lo explican ex post -si es que finalmente logran sobreponerse a él-como tal purificación inscrita en un plan divino. Un problema que tiene esta estrategia es que exige poner en cuestión de manera global las tradiciones religiosas en las que se inscribe el fenómeno. Ni Teresa de Ávila, ni Juan de la Cruz, ni Teresa de Calcuta deberían ser tomados como ejemplos de vida de fe para nosotros. Al seguir esta estrategia de rebajar la importancia del fenómeno se estaría sugiriendo que el ideal del seguimiento de Cristo tal como lo han vivido los santos es un extremo patológico que conduce, paradójicamente, a la pérdida de una característica considerada central de la vida cristiana: la fe. Una teoría que pretende explicar la naturaleza de la fe desde una perspectiva que se toma en serio la experiencia de vida de los cristianos, no puede suponer como una consecuencia razonable de tal teoría que las personas que -según los parámetros internos de la tradición religiosa- han sido un ejemplo de vida cristiana, no han tenido fe. Swinburne, por lo pronto, no tiene ninguna intención de involucrarse en un programa de crítica tan radical. Tampoco lo tiene Bishop ni, por supuesto, el Magisterio de la Iglesia Católica. Lo razonable es suponer, por el contrario, que personas como san Juan de la Cruz, santa Teresa de Ávila y la beata Teresa de Calcuta son personas de fe.

Una segunda estrategia sería sostener que los sujetos que se encuentran enfrentados a una noche oscura, a pesar de las apariencias en contrario, sí saben que tienen fe. La única forma de acomodar un alegato de este tipo para explicar el fenómeno sería sostener que el sujeto que está padeciendo una noche oscura del alma tiene fe, sabe que tiene fe, pero no sabe que sabe que tiene fe. Por supuesto, un caso de este estilo no sería extraño para una concepción externalista acerca de la fe en donde uno puede saber algo y no saber que uno sabe algo, pero no lo es en una concepción internalista. Se consigue el conocimiento de la propia fe, pero al precio del desconocimiento del conocimiento de la propia fe, lo que no es coherente con el internalismo. En segundo lugar, se trataría de una explicación muy poco creíble. La estructura de un estado mental de este tipo sería bastante peculiar, en efecto, con tres niveles sobrepuestos diferentes. El único 
motivo para postular tal estructura sería la pretensión de mantener - en lo que cabe- las exigencias internalistas, pues es mucho más simple postular que no se conoce que uno tiene fe. Presentar una explicación de este tipo como la forma de acomodar el fenómeno de la noche oscura a un esquema internalista sería, por lo tanto, petición de principio.

Entonces, no es sencillo explicar el fenómeno de la noche oscura en una teoría internalista de la fe. Esto afecta, tal como se ha indicado, a la concepción de Swinburne, pero en los casos de la concepción católica y de las teorías no-cognitivistas el impacto del fenómeno de la noche oscura del alma requiere una consideración especial.

\subsection{Internalismo acerca del testimonio y la teoría católica de la fe}

En la teoría católica, el acto de fe sobrenatural es el acto por el que se asiente a la verdad revelada por Dios, debido a la autoridad de Dios que revela, quien no puede engañarse ni engañarnos. Este acto de asentimiento ha de tener por objeto una proposición, que es lo revelado por Dios. El valor epistemológico de la fe depende de la concepción general que se tenga acerca del valor epistemológico del testimonio. De un modo completamente general, uno está justificado en aceptar como verdadera una proposición, sea $p$, por testimonio si y sólo si hay alguien, sea $S$, que ha aseverado que $p$, conoce que $p$ y es veraz al aseverar que $p$. Tal como se ha indicado más arriba, lo que diferencia a la fe sobrenatural de otros casos de testimonio es el carácter peculiar del testigo. Si la fe sobrenatural ha de tener algún valor epistemológico es porque: (i) de un modo general, el testimonio es aceptable como una forma de justificación de nuestras creencias, y (ii) la revelación divina es una forma cualificada de testimonio que merece nuestro asentimiento. Si se atiende a la discusión acerca del valor epistemológico del testimonio se han presentado dos grandes opciones sistemáticas: el reductivismo acerca de la fe y el anti-reductivismo acerca de la fe. Las teorías reductivistas han sido típicamente internalistas. Las teorías anti-reductivistas pueden ser tanto internalistas como externalistas. El valor epistemológico de 
la fe sobrenatural en la teoría católica de la fe depende de un modo directo de qué teoría general del testimonio se acepte.

En una concepción reductivista, el valor epistemológico del testimonio se reduce a otras fuentes de justificación y conocimiento. ${ }^{4}$ Lo que se espera aquí de un sujeto racional para que llegue a estar justificado en creer aquello de que se le ha dado testimonio es que haga una inferencia de la que se siga que el testimonio es epistemológicamente confiable desde premisas que no están -a su vez- justificadas por testimonio. Esto es, se espera que el sujeto efectúe una ponderación racional del testimonio de acuerdo con la evidencia que se posea. De un modo general, se puede sostener que $S_{1}$ está justificado en creer que $p$ por el testimonio que $S_{2}$ ha dado de $p$, si y sólo si $S_{1}$ conoce o tiene evidencia suficiente para creer que:

(i) $S_{2}$ ha aseverado que $p$ [premisa de contenido];

(ii) Si $S_{2}$ ha aseverado que $p$, entonces $S_{2}$ cree que $p$ [premisa de veracidad]; y

(iii) Si $S_{2}$ cree que $p$, entonces $S_{2}$ está justificado en creer que $p$ [premisa de confiabilidad epistemológica].

Por aplicación reiterada de modus ponens sobre (i)-(iii) se sigue que $S_{2}$ está justificado en creer que $p$. Si $S_{1}$ tiene evidencia suficiente para creer (i)-(iii), entonces tiene evidencia suficiente para creer lo que se sigue de (i)-(iii). Quienquiera que esté justificado en creer que $S_{2}$ está justificado en creer que $p$, está también justificado en creer que $p$. Es crucial en una concepción reductivista que la evidencia que posea el testigo para la proposición de que da testimonio debe

4 El testimonio por sí mismo no aumenta la evidencia para una proposición, lo más que se puede esperar de él es que permita preservar la evidencia que el testigo posee para la proposición de que está dando testimonio. Una fuente de justificación puede ser derivativa en este sentido y, sin embargo, no ser reducible a otras. La memoria, por ejemplo, también es un mecanismo que permite preservar la evidencia que se ha obtenido en el pasado por otras fuentes. Nadie podría, sin embargo, pretender dispensarse de ella. No habría modo de transmitir la evidencia obtenida en el pasado sin su concurrencia. La memoria es, entonces, una fuente de justificación derivativa sin ser reducible. 
ser ponderada por la evidencia que tenga quien reciba tal testimonio acerca de la veracidad del testigo y su confiabilidad epistemológica. Para efectuar esta ponderación, quien recibe el testimonio debe recabar evidencia empírica para justificar las premisas del tipo (i)-(iii). Obligatoriamente, entonces, la evidencia que posea el testigo va a verse sustancialmente reducida en cada cadena de transmisión.

El reductivismo ha sido criticado porque resulta demasiado exigente epistemológicamente e impide explicar la confianza, que existe tanto en la vida diaria como en el desarrollo de la ciencia, para nuestras prácticas epistémicas. La ciencia natural es una empresa cooperativa de carácter social que descansa en la fiabilidad del testimonio. Es muy dudoso que una teoría reductivista pueda reconstruir tal fiabilidad. No es necesario, de todos modos, discutir aquí esta cuestión, pues tanto el reductivismo como su alternativa internalista estarán afectadas por el mismo problema. Quienes rechazan el reductivismo, sin embargo, están inclinados a pensar que la justificación que uno tenga para una creencia debe ser algo disponible internamente para el sujeto. Han sostenido que el valor epistemológico del testimonio viene dado por un principio defectible de este tipo:

(*) Si $S_{2}$ da testimonio a $S_{1}$ de que $p$, entonces, en condiciones normales, es racional para $S_{1}$ creer que $p$ debido al testimonio de $S_{2}$, a menos que $S_{1}$ tenga razones especiales para objetar la confiabilidad o la veracidad de $S_{2}$.

Este principio (*) es defectible pues, aunque legitima para aceptar el testimonio que a uno se le entregue, obliga a efectuar una ponderación tal como la pide el reductivista si es que hay razones especiales y positivas para dudar de la veracidad o de la confiabilidad del testigo. No existiendo tales razones positivas que puedan desvirtuar la operación del principio (*), uno simplemente debe aceptar lo que se ha aseverado. En un caso de este tipo la evidencia que poseía el testigo se preserva íntegra. Todo esto debe ser accesible para el sujeto desde su perspectiva interna.

Una concepción católica de la fe podría - al menos en abstracto- complementarse con una explicación reductivista del valor del 
testimonio o con una explicación internalista que postule un principio defectible tal como (*). En el primer caso, tener fe es aceptar el testimonio de Dios habiendo ponderado qué tan verosímil es que exista una entidad como Dios, que sea veraz, que sea omnisciente y que haya revelado lo que se supone que ha revelado. Por supuesto, si la fe ha de tener valor epistemológico, entonces esta ponderación debería arrojar una probabilidad epistémica superior a 0,5. En cualquier caso, si el creyente no es irracional al tener fe, la probabilidad subjetiva ${ }^{5}$ que debería asignarle a esta ponderación debería ser superior a 0,5. Tener fe no es simplemente el acto de aceptar la revelación divina, sino que es tal aceptación unida a una ponderación racional acerca de la calidad del testimonio que se ha ofrecido, accesible -naturalmente- para el creyente. En el segundo caso, tener fe es aceptar el testimonio de Dios habiendo aplicado un principio defectible tal como el principio (*) con la conciencia de no haber desvirtuadores para su aplicación. Para el creyente, entonces, debe resultar accesible tanto su aceptación, como el principio (*), como la evaluación epistemológica acerca de la presencia o ausencia de desvirtuadores. Si un creyente no es irracional debería, por lo menos, tener la convicción interna de que no hay desvirtuadores, aunque esta convicción sea errada.

Ninguna de estas dos alternativas funciona muy bien ante el fenómeno de la noche oscura. Un creyente al que no se pueda atribuir completa irracionalidad debería tener la convicción interna, o bien de que la ponderación es positiva, o bien de que no hay desvirtuadores para el principio defectible (*). Nada de esto se le podría atribuir a quien se encuentra en una etapa de noche oscura del alma. La aceptación de la revelación divina en casos de este tipo es "ciega". En algunos casos hasta la mera aceptación se torna oscura para el fiel. Alguien sometido a una noche oscura no posee ninguna convicción subjetiva -que le resulte disponible internamente- de que hay

5 La probabilidad subjetiva que un sujeto le asigna a una proposición es una función que representa el grado de 'firmeza' con que el sujeto cree tal proposición mapeando cada proposición a un número real entre o y 1. La probabilidad epistémica, en cambio, es el grado de aceptación que se le debe dar a la proposición en cuestión dada la evidencia existente. 
una ponderación positiva del valor epistemológico de la revelación divina, o de que no hay desvirtuadores para aplicar un principio defectible. Si estos son elementos constitutivos de un acto de fe, nadie que esté pasando por una noche oscura posee fe.

\subsection{La noche oscura y la "empresa doxástica”}

En la concepción no-cognitivista defendida por Bishop la fe es una "empresa doxástica" (doxastic venture). Esto es, se trata del acto de asumir el riesgo de actuar como si una proposición fuese verdadera, aunque no exista evidencia suficiente para creer que sea verdadera. En la teoría de Bishop - que pretende ser una elaboración de la propuesta pragmatista de William James- el creyente no está actuando de manera irracional al embarcarse en tal empresa doxástica, pues no se hace en contra de la evidencia para la proposición o proposiciones de que se trata. Por otro lado, asumir este riesgo es razonable prácticamente, pues hay ganancias en la propia vida que compensan el riesgo que se está tomando. Esto parece un "cálculo estratégico", pero en la concepción de Bishop el motor fundamental para asumir una empresa doxástica es afectivo y emocional. ${ }^{6}$

El fenómeno de la noche oscura es problemático para una teoría de este tipo por varios motivos. En primer lugar, es característico de la teoría de la empresa doxástica que no hay para el creyente

6 Uno podría estar inclinado a identificar la propuesta de Bishop con la apuesta de Pascal (Pensamientos, III, §233), según la cual la creencia en la verdad del cristianismo se devela como más racional que su no creencia en virtud las ganancias o beneficios que supondría la efectiva existencia de Dios para el hombre. Si Dios existe, entonces el creyente gana la vida eterna, si no existe, no gana nada. Empero, si Dios existe, el no creyente no gana nada y si no existe tampoco. Ante la ausencia de evidencia a favor o en contra de la existencia de Dios, es conveniente actuar como si Dios existiese. Pese a que la empresa doxástica y la apuesta sean muy semejantes en ciertos aspectos, lo cierto es que en el caso de Bishop el elemento decisivo para la creencia está dado por la afectividad y no por el cálculo racional de beneficios. De hecho, el énfasis está puesto en que la creencia en la verdad cristiana no es irracional más que en la prueba de que es racional. Por otro lado, las ventajas prácticas derivadas de la fe que reconoce Bishop están inscritas en el horizonte mundano o inmanente más que en el trascendente, es decir, se tiene en cuenta más bien la calidad de la vida terrenal que la posible salvación o condena trasmundana. 
evidencia a favor o en contra de las proposiciones de que se trate. La fe es una actitud que sólo se produce en una situación de oscuridad evidencial. Pero esto es exactamente lo que sucede en una situación de noche oscura. El fiel no tiene claridad sobre la evidencia que posee y le parece, además, que no hay ninguna evidencia. Lo extraño es que, según los defensores del punto de vista no-cognitivista, del que la teoría de la empresa doxástica es una representante característica, esto debería ser la situación normal. Todos los fieles se deberían encontrar en la misma situación de oscuridad epistémica, pues eso es lo propio de, en efecto, tener fe por oposición a otras formas de actitud proposicional. No tiene, entonces, ningún sentido que exista un fenómeno de purificación interior de ocurrencia excepcional para personas de gran vida interior que consista en tal oscurecimiento. No tiene ningún sentido que quienes padecen una noche oscura $p a-$ dezcan por ella. Si se quiere, la situación que se produce aquí respecto del fenómeno de la noche oscura es el inverso a la situación que se produce en las teorías cognitivistas internalistas. En las teorías cognitivistas internalistas no debería suceder algo así como una noche oscura. No debería suceder que alguien que tuviese una fe profunda estuviese en una situación de opacidad interior respecto de su propia fe. Desde la perspectiva de las teorías no-cognitivistas, por el contrario, no debería suceder que la situación de la noche oscura fuese excepcional, pues es lo que debería esperarse para el principiante en la vida interior y para el fiel común y corriente.

Sería más razonable sostener que la "noche oscura" debería re-describirse en una teoría no-cognitivista como el oscurecimiento respecto de la ponderación interior de los riesgos que se asumen con la empresa doxástica, respecto de la ponderación interior de las ventajas que se pueden alcanzar con tal empresa y respecto de la resolución interior a emprender. El fenómeno de la noche oscura debería ser entendido como una situación en donde uno llega a no tener claro si "realmente vale la pena" la empresa asumida. Pero esto no tiene mucho que ver con el fenómeno, tal como éste ha sido reportado. Quienes padecen una noche oscura del alma tienen una profunda sed de Dios. En gran parte, esto es lo que hace que su situación de oscuridad interior resulte tan dolorosa y purificadora. No es 
la situación de "pérdida de entusiasmo" en una empresa porque uno advierte riesgos crecientes. Es una situación en donde uno preserva la resolución interior de buscar a Dios por sobre todas las cosas. Lo que sucede es que Dios parece ocultarse.

\section{Externalismo y el valor epistemológico de la fe}

Tal como se ha mostrado, entonces, ni las teorías cognitivistas de corte internalista, ni las teorías no-cognitivistas, parecen acomodarse bien al fenómeno de la noche oscura del alma. Éste es un motivo que obliga a considerar alternativas, en primer lugar, externalistas y, en segundo lugar, en donde la noche oscura es un fenómeno de oscurecimiento cognitivo. Esto es, el fenómeno de la noche oscura debería poder ser entendido como una situación en donde alguien tiene fe pero llega a no saber que tiene fe y, además, es un fenómeno donde lo que se oscurece interiormente para el fiel es el valor epistemológico de la fe. Sólo una concepción de la fe que respete su carácter cognitivo y en donde se haga espacio para la opacidad interior puede explicar cómo suceden casos de noche oscura para los santos.

Una alternativa de este tipo es la que ofrece, por supuesto, la epistemología reformada, pues aquí se caracteriza la fe como una creencia básica dotada de garantía, y cuya garantía epistemológica proviene de la operación de facultades cognitivas o mecanismos cognitivos funcionando adecuadamente en el ambiente para el que han sido diseñadas: el sensus divinitatis y la acción directa del Espíritu Santo sobre la mente del fiel. No se requiere, de manera adicional, que el fiel conozca o tenga creencias bien justificadas y accesibles internamente acerca de la adecuación del funcionamiento del sensus divinitatis y de la acción del Espíritu Santo. Basta con que tales mecanismos estén objetivamente funcionando de manera adecuada para que se posean creencias con garantía epistemológica. Para alguien, entonces, puede acaecer que, de serle internamente patente que posee tal facultad o que está operando el Espíritu Santo, se pasa a una situación de oscuridad en que la propia fe resulte opaca. 
No sólo, sin embargo, es la epistemología reformada la que ofrece una concepción cognitivista y externalista de la fe apta para explicar cómo puede acaecer una noche oscura del alma. También la teoría católica ofrece una concepción cognitivista y externalista de la fe, si es que el valor epistemológico del testimonio es explicado del modo apropiado. Tal como se ha indicado más arriba, en la concepción católica la fe es el acto de aceptar como verdaderas las proposiciones reveladas por Dios. El valor epistemológico que pueda concederse a la fe tiene que ver con el valor epistemológico que pueda concederse al testimonio. Hay, por supuesto, teorías internalistas para explicar el valor epistemológico del testimonio, tal como se explicó más arriba: el reductivismo acerca del testimonio y la concepción en donde éste depende de la aplicación reflexiva de un principio defectible. Cuando se integran esas dos concepciones para la conformación de una teoría de la fe sobrenatural, ninguna de ellas parece funcionar muy bien para explicar cómo es que se puede producir un fenómeno de noche oscura del alma. Existe también, sin embargo, una explicación externalista del valor epistemológico del testimonio (Goldberg, 2010; Zagzebski, 2012). En una concepción externalista del testimonio si alguien, sea $S_{1}$ acepta que $p$ debido al testimonio que $S_{2}$ ha dado de $p$, y $S_{2}$ está justificado en creer que $p$, entonces $S_{1}$ está justificado en creer que $p$. Del mismo modo, si $S_{2}$ conoce que $p$ y $S_{1}$ acepta el testimonio de $S_{2}$ acerca de $p$, entonces $S_{1}$ conoce que $p$. Lo único que se requiere para que el testimonio sea una fuente de justificación y de conocimiento es que la fuente de la que emana - el testigo- sea objetivamente veraz y tenga conocimiento o justificación. No se requiere que $S_{1}$ tenga conocimiento ulterior de que $S_{2}$ es veraz al dar testimonio de que $p$, ni de que $S_{2}$ está justificado en creer que $p$. Basta con que $S_{2}$ sea veraz y esté justificado. Tampoco se requiere, tal como en las concepciones internalistas, que $S_{1}$, quien recibe el testimonio, consciente y reflexivamente esté aplicando una regla defectible tal como (*) al testimonio que recibe. Puede ser que su conducta se acomode a una regla como $(*)$, pero no se requiere que él conozca que su conducta se conforma con la regla $(*)$. Es obvio que, desde esta perspectiva externalista del testimonio, la fe sobrenatural es conocimiento. Dios es omnisciente y máximamente veraz, de modo que no 
puede engañarse ni engañarnos. Es necesario que si Dios cree que $p$, entonces $p$. Es necesario que si $p$, entonces Dios cree que $p$. La perfección moral de Dios hace imposible, por otro lado, que pueda estar pretendiendo engañarnos al hacernos creer algo. Dios no es un malin génie. Si $S$ llega a creer que $p$ debido a la revelación que ha hecho Dios de que $p$, entonces $S$ conoce que $p$. La evidencia que posee Dios para la proposición que revela se transmite íntegra.

Cuando se integra una concepción de este tipo para la conformación de una teoría de la fe sobrenatural, resulta posible que para alguien llegue a resultar opaca interiormente la propia fe que se posee. Esto no impide que el fiel tenga fe y, lo que es más, que tal fe tenga valor epistemológico y sea conocimiento. De un modo análogo a como se producen fenómenos de oscurecimiento interior, tal como pasa en la noche oscura del alma, también -correlativamentepueden darse fenómenos de "iluminación interior" de la propia fe. Algo de este estilo es lo que cabe esperar que suceda cuando alguien pasa de tener una fe no muy reflexiva a una fe epistemológicamente madura y consciente. Una concepción cognitivista y externalista de la fe hace posible la ocurrencia de fenómenos de este tipo. Es posible que un niño pequeño con pocas capacidades reflexivas tenga fe sobrenatural y, con ello, conocimiento. Luego esta fe puede verse fortalecida al corroborar reflexivamente sus credenciales epistemológicas. Para un internalista, en cambio, no hay cómo acomodar la fe de niños y creyentes epistemológicamente poco sofisticados. Para el internalista ellos no tienen fe, o su fe no cuenta como conocimiento. De un modo semejante, alguien de gran vida interior puede llegar a sufrir un oscurecimiento interior profundamente purificador en que esta misma fe le llegue a resultar opaca. Esto no impide que tenga fe y, con ello, conocimiento.

\section{Conclusiones}

Se ha presentado en este trabajo el problema que plantean los fenómenos místicos de la "noche oscura del alma" para teorías internalistas de la fe sobrenatural. En teorías de este tipo la fe sobrenatural, 
como estado subjetivo, debe ser algo inmediatamente patente para quien la posea desde su perspectiva de primera persona. Esto es lo que sucede en teorías como la defendida recientemente por Swinburne y también en varias formas de interpretar la concepción católica tradicional de la fe sobrenatural. El valor epistemológico que pueda tener la fe, ya sea como una forma de conocimiento o de justificación, depende de que esté accesible internamente la evidencia para el sujeto desde su perspectiva interna de primera persona. Los fenómenos asociados con la "noche oscura del alma", sin embargo, parecen ser incompatibles con esta descripción. Si uno aceptase la concepción internalista, estos deberían ser tratados como casos en que sencillamente se ha perdido la fe. Tampoco parecen explicar bien el fenómeno de la noche oscura del alma las teorías no cognitivistas de la fe, tal como la teoría de la "empresa doxástica”. En estos casos, se hace inexplicable que sea penosa una noche oscura del alma, pues debería ser la situación normal para cualquier fiel.

Las teorías que parecen explicar bien cómo es que puede ocurrir un fenómeno de este tipo son las teorías cognitivistas y externalistas. En un modelo externalista de la fe sobrenatural estos fenómenos de oscurecimiento son explicables. No es necesario que uno conozca que posee un estado mental para poseerlo. Es más, no es necesario que uno posea conocimiento de que uno posee conocimiento para tener conocimiento. La fe sobrenatural puede ser admitida como una forma de conocimiento incluso en quienes no tienen la suficiente capacidad reflexiva como para considerar críticamente la calidad de la evidencia que poseen, o que - tal como sucede en los fenómenos de la noche oscura- se encuentran en un profundo proceso de purificación interna en donde su propia fe, esperanza y caridad quedan envueltas en sombras de duda. ${ }^{7}$

7 Este proyecto ha sido redactado en ejecución del proyecto de investigación VRIPastoral № 1565/DPCC2012 de la Vice-Rectoría de Investigación de la Pontificia Universidad Católica de Chile. Una versión preliminar fue presentada en las XIII Jornadas de Filosofía, "Fe, Razón y Cultura: un diálogo necesario desde la filosofía", Universidad de la Santísima Concepción, Concepción, 28 y 29 de agosto de 2013. Agradecemos los comentarios y sugerencias de los asistentes a estas Jornadas. 


\section{REFERENCIAS BIBLIOGRÁFICAS}

Alston, William P. 1993. Perceiving God. The Epistemology of Religious Experience. Ithaca: Cornell University Press.

BisHOP, John. 2007. Believing by Faith. An Essay in the Epistemology and Ethics of Religious Belief. Oxford: Clarendon Press.

BisHOP, John. 2010. “Faith” en Ed Zalta (ed.) Stanford Encyclopedia of Philosophy, http: / / plato.stanford.edu/entries/faith/. Obtenido el 28 de diciembre de 2013.

Bonjour, Laurence. 2002. "Internalism and Externalism" en Paul K. Moser (ed.) The Oxford Handbook of Epistemology. Oxford: Oxford University Press, pp. 234263.

Denzinger, Heinrich y Peter Hünermann (eds.) (DH). 1999. El magisterio de la Iglesia. Enchiridion symbolorum definitionum et declarationibus de rebus fidei et morum. Barcelona: Herder.

Francisco, Papa. 2013. Lumen fidei, carta encíclica. Santiago: Ediciones UC.

Garrigou-Lagrange, Réginald. 1944. Las tres edades de la vida interior. Buenos Aires: Desclée.

Goldberg, Sanford C. 2010. Relying on Others. An Essay in Epistemology, Oxford: Oxford University Press.

Goldman, Alvin. 1999. "Internalism Exposed" en The Journal of Philosophy, 96, pp. 271-293. Reimpreso en Ernest Sosa, Jaegwon Kim, Jeremy Fantl y Matthew McGrath (eds.), Epistemology. An Anthology, Oxford., Blackwell, 2008, pp. 379 393.

Howard-Snyder, Daniel y Paul K. Moser (eds.) 2002. Divine Hiddenness. New Essays, Cambridge: Cambridge University Press.

Juan de la Cruz, san. 1994. "Poesías" en Obras completas. Madrid: Biblioteca de Autores Cristianos.

Moser, Paul K. 2008. The Elusive God. Reorienting Religious Epistemology, Cambridge: Cambridge University Press.

Plantinga, Alvin. 1983. "Reason and Belief in God" en Alvin Plantinga y Nicholas Wolterstorff (eds.), Faith and Rationality. Reason and Belief in God. Notre Dame: University of Notre Dame Press.

Plantinga, Alvin. 1993a. Warrant: The Current Debate. Oxford: Oxford University Press.

Plantinga, Alvin. 1993b. Warrant and Proper Function. Oxford: Oxford University Press.

Plantinga, Alvin. 2000. Warranted Christian Belief. Oxford: Oxford University Press. 
Swinburne, Richard. 2001. Epistemic Justification. Oxford: Clarendon Press.

Swinburne, Richard. 2004. The Existence of God. Oxford: Clarendon Press (2da. edición).

Swinburne, Richard. 2005. Faith and Reason. Oxford: Clarendon Press (2da. edición).

TANQuerey, Adolphe. 1930. Compendio de teología ascética y mística. Paris: Desclée.

Teresa de Ávila, Santa. 1941. “¡Cuán triste es, Dios mío!” en Obras de Santa Teresa de Jesús, Buenos Aires., Editorial Poblet.

Teresa de Calcuta, Beata. 2008. Madre Teresa: ven, sé mi luz. Las cartas privadas de la santa de Calcuta. Editadas por Brian Kolodiejchuk. Barcelona: Editorial Planeta. Traducción de Pablo Cervera.

TomÁs de Aquino, Santo. 1952. Summa theologiae. Roma: Marietti.

Williamson, Timothy. 2000. Knowledge and its Limits. Oxford: Oxford University Press.

Zagzebski, Linda T. 2012. Epistemic Authority. A Theory of Trust, Authority, and Autonomy in Belief. Oxford: Oxford University Press. 